\title{
BMJ Open Development of the Serious Illness Care Program: a randomised controlled trial of a palliative care communication intervention
}

\author{
Rachelle Bernacki, ${ }^{1,2,3,4}$ Mathilde Hutchings, ${ }^{2,3}$ Judith Vick, ${ }^{5}$ Grant Smith, ${ }^{6}$ \\ Joanna Paladino, ${ }^{2,3}$ Stuart Lipsitz, ${ }^{3}$ Atul A Gawande, ${ }^{1,3}$ Susan D Block ${ }^{1,2,3,4}$
}

To cite: Bernacki R, Hutchings M, Vick J, et al. Development of the Serious Illness Care Program: a randomised controlled trial of a palliative care communication intervention. BMJ Open 2015;5:e009032. doi:10.1136/bmjopen-2015009032

- Prepublication history for this paper is available online. To view these files please visit the journal online (http://dx.doi.org/10.1136/ bmjopen-2015-009032).

Received 9 June 2015 Revised 3 September 2015 Accepted 14 September 2015

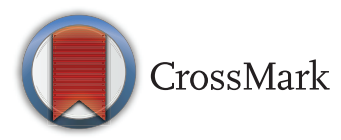

For numbered affiliations see end of article.

Correspondence to Dr Rachelle Bernacki; rbernacki@partners.org

\section{ABSTRACT}

Introduction: Ensuring that patients receive care that is consistent with their goals and values is a critical component of high-quality care. This article describes the protocol for a cluster randomised controlled trial of a multicomponent, structured communication intervention.

Methods and analysis: Patients with advanced, incurable cancer and life expectancy of $<12$ months will participate together with their surrogate. Clinicians are enrolled and randomised either to usual care or the intervention. The Serious IIIness Care Program is a multicomponent, structured communication intervention designed to identify patients, train clinicians to use a structured guide for advanced care planning discussion with patients, 'trigger' clinicians to have conversations, prepare patients and families for the conversation, and document outcomes of the discussion in a structured format in the electronic medical record. Clinician satisfaction with the intervention, confidence and attitudes will be assessed before and after the intervention. Self-report data will be collected from patients and surrogates approximately every 2 months up to 2 years or until the patient's death; patient medical records will be examined at the close of the study. Analyses will examine the impact of the intervention on the patient receipt of goal-concordant care, and peacefulness at the end of life. Secondary outcomes include patient anxiety, depression, quality of life, therapeutic alliance, quality of communication, and quality of dying and death. Key process measures include frequency, timing and quality of documented conversations.

Ethics and dissemination: This study was approved by the Dana-Farber Cancer Institute Institutional Review Board. Results will be reported in peer-reviewed publications and conference presentations.

Trial registration number: Protocol identifier NCT01786811; Pre-results.

\section{INTRODUCTION}

Ensuring that patients receive care that is consistent with their goals and values is a critical component of high-quality care. ${ }^{1}$ Early conversations about advance care planning (ACP) with seriously ill patients have been associated with better outcomes for patients and families. ${ }^{2-4}$ However, multiple deficits in the timing and content of these discussions have been described. Discussions occur too late, when patients are in crisis or unable to make decisions for themselves, or clinicians who know the patient are not available. ${ }^{5-7}$ Even though most patients want to know about their prognosis, such information is often not shared, ${ }^{8}$ leading to poorly informed decisions. Furthermore, contrary to expert recommendations, ${ }^{1}$ physicians tend to focus these critical conversations on choices about procedures (eg, resuscitation or feeding tube insertion) rather than on the goals, values and wishes that form the basis of an informed patient's decisions. Clinicians are underprepared and undertrained to conduct high-quality end-of-life conversations, ${ }^{10}$ and tend to avoid them. ${ }^{11}$ Several studies of physicians in the UK, ${ }^{12}$ the $\mathrm{USA}^{13}$ and in an international palliative care training programme ${ }^{14}{ }^{15}$ demonstrate that communication skills instruction programmes are effective, ${ }^{16}$ with participants showing sustained improvement in patientcentered communication skills, including significant improvement in responses to patients' emotional cues. ${ }^{12}$ Interactive, casebased learning sessions with communication skills practice are the most effective $;{ }^{17}$ however, most often, these training programmes have been intensive, multiday off-site retreats which are not always feasible for busy clinicians in practice.

One proposed solution to deficits in ACP and end-of-life discussions is for palliative care clinicians, who are trained to conduct such conversations, to see all seriously ill 
patients. Early palliative care intervention, the primary focus of which is communication, ${ }^{18}$ with ambulatory patients with non-small cell lung cancer has been demonstrated to improve quality of life, mood and survival, and to reduce aggressiveness of care and hospital length of stay. ${ }^{4}$ While palliative care physicians are an excellent resource for conducting/assisting with end-of-life conversations, there is already a shortage of approximately 11000 palliative medicine physicians for hospices and hospital-based palliative care programmes; ${ }^{19}$ this workforce shortage is only expected to increase with future demographic changes. It is unlikely that this care model will be scalable. ${ }^{20}$ Thus, other approaches are needed, to engage non-palliative care physicians in integrating basic elements of palliative care, such as ACP, in the care of patients with serious illnesses.

We have developed, piloted and refined a multicomponent Serious Illness Care Program (SICP) to improve conversations about values and goals for patients with serious illnesses. The goals of this programme are to train and support oncology clinicians in integrating best practices in ACP in the care of their patients, to optimise the alignment between patient goals and the medical care they receive, and to enhance quality of life and peacefulness throughout the late stages of illness. We describe the steps taken in developing the programme, including review of the evidence about the effectiveness of ACP discussions, ${ }^{21} 22$ pilot work with oncologists to improve their ACP practices, engagement of a national advisory board, and development and pilot-testing of a structured approach to these conversations in the form of a seven-item Serious Illness Conversation Guide (SICG) for clinicians and patients. Then, we describe the research protocol: setting, recruitment, measurements, design of the intervention, timing of assessments, data analysis and collection, and planned analyses and dissemination.

\section{Literature review}

We conducted and published an extensive literature review of the evidence about early goals-of-care discussions. ${ }^{21}$ These discussions were found to be associated with better quality of life, reduced utilisation of non-beneficial medical care near death, enhanced goal-consistent care, positive family outcomes and reduced costs. Existing evidence does not support the commonly held belief that communication about end-of-life issues increases patient distress. The review also described best practices in conversations about serious illness care goals, and offered guidance to clinicians and healthcare systems on following a systematic approach to quality and timing of such communication to assure that each patient has a personalised serious illness care plan.

\section{Pilot work}

To begin understanding how the physicians in our institution actually conduct ACP discussions, we asked oncologists in the Gastrointestinal Oncology and Neuro-oncology centres at the Dana-Farber Cancer Institute (DFCI) to conduct values and goals discussions with their patients with pancreatic cancer or glioblastoma multiforme as part of an institutional quality improvement initiative. We evaluated clinical documentation before and after education where we introduced clinicians to an ACP module in the electronic medical record (EMR) intended to simplify tracking of patient values and goals. The proportion of patients whose physician documented their values and goals was low $(<5 \%)$ before the intervention, and the norm $(>90 \%)$ after the intervention. Following this pilot initiative, we solicited feedback from the clinicians with a structured interview about their experiences in conducting and documenting these discussions. This feedback later informed the development of our conversation guide and training programme.

\section{Consultation with National Advisory Group}

Early in the project, we gathered a national panel of experts for an Advisory Group for the SICP. Members included palliative care specialists, general internists, cardiologists, oncologists, paediatricians, surgeons and patients. Some members had extensive expertise in end-of-life communication skills and ACP; others were representative clinicians and patients who would be using the intervention. The purpose of the meeting was to discuss key elements of agreement and disagreement about best practices in serious illness communication, to elicit feedback about a proposed intervention, and to obtain input on our research design. Prior to the meeting, all members received an extensive background document, including a literature review.

\section{Development of the SICP materials}

Building on our literature review and pilot work, we developed a systematic approach to integrate best practices in outpatient care for patients with serious illnesses, and proposed a structured format to guide the discussions-the novel seven-item SICG (figure 1). This guide addresses eliciting illness understanding, eliciting decision-making preferences, sharing prognostic information according to preferences, understanding goals and fears, exploring views on trade-offs and impaired function, and wishes for family involvement. In addition to the SICG, designed for clinicians to use in outpatient encounters with their patients, we developed a letter to give patients before the visit to help them prepare for the discussion, and a family communication guide to send home with patients after the discussion to aid in discussing their values and goals with their family. The family guide follows the same general structure as the SICG, but in the patient's voice.

\section{Patient input}

We sought feedback from the DFCI Patient and Family Advisory Council about our materials (SICG, family 
Figure 1 Serious IIIness Conversation Guide.

\section{Serious Illness Conversation Guide}

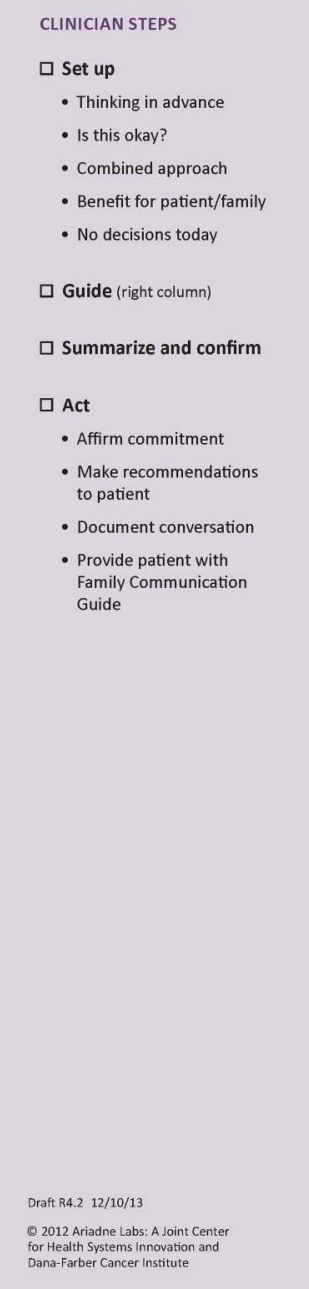

CONVERSATION GUIDE

Understanding What is your understanding now of where you are with your illness?

\begin{tabular}{|c|c|}
\hline $\begin{array}{l}\text { Information } \\
\text { preferences }\end{array}$ & $\begin{array}{l}\text { How much information about what is likely to be } \\
\text { ahead with your illness would you like from me? } \\
\text { FOR EXAmpLE: } \\
\text { Some patients like to know about time, others like to know } \\
\text { what to expect, others like to know both. }\end{array}$ \\
\hline Prognosis & Share prognosis, tailored to information preferences \\
\hline Soals & $\begin{array}{l}\text { If your health situation worsens, what are your } \\
\text { most important goals? }\end{array}$ \\
\hline
\end{tabular}

\begin{tabular}{ll}
$\begin{array}{l}\text { Fears / } \\
\text { Worries }\end{array}$ & $\begin{array}{l}\text { What are your biggest fears and worries about } \\
\text { the future with your health? }\end{array}$ \\
\hline Function & $\begin{array}{l}\text { What abilities are so critical to your life that } \\
\text { you can't imagine living without them? }\end{array}$ \\
\hline Trade-offs & $\begin{array}{l}\text { If you become sicker, how much are you } \\
\text { willing to go through for the possibility of } \\
\text { gaining more time? }\end{array}$
\end{tabular}

Family How much does your family know about your priorities and wishes?

(Suggest bringing family and/or health care agent to next visit to discuss together) guide, pre-visit letter, etc) through a series of meetings. The Patient and Family Advisory Council consists of patients, family members, executive leaders and providers partnering to improve hospital programmes, policies and the overall quality of care of the DFCI. In addition, we worked with a marketing firm (Hill Holliday, http://www.hhcc.com) that conducted patient, family and clinician focus groups to determine acceptability of terminology, wording and format; we used these data to refine our materials.

\section{Pilot testing the guide}

We piloted the SICG with 26 practitioners-physicians from multiple specialties including internal medicine, oncology, nephrology, critical care, surgery and neurology, as well as nurse practitioners (NPs) and physician's assistants. Clinicians were given a brief overview of the project and were trained to use the conversation guide. They were then observed and audio-recorded as they conducted a discussion with a trained actor serving as a standardised patient. Clinicians first followed their usual approach to an end-of-life conversation, then were asked to use the guide's structured format. We then debriefed with the clinicians about the wording, order, structure and acceptability of the guide, as well as any other feedback. We made changes based on clinicians' suggestions. We used the newly edited version of the SICG for the next round of interviews. We continued this cycle (approximately 3 clinicians per cycle) until our participants suggested no more significant changes to the guide (eight cycles).

\section{METHODS AND ANALYSIS \\ Trial design}

The study is a prospective, cluster-randomised controlled trial of a quality-improvement intervention. We enrol oncology physicians, NPs, physician assistants (PAs) and ambulatory patients with cancer and their identified surrogates. We are conducting the study at the DFCI, and at two affiliated satellite clinics (Dana-Farber at Milford Regional Medical Center, and Dana-Farber at South 
Shore Hospital). DFCI is a National Cancer Institute designated comprehensive cancer centre located in Boston, Massachusetts and part of the National Comprehensive Cancer Network. The DFCI is organised into 'disease centres', each of which focuses on a specific class of illnesses. Clinicians from these disease centres, except for those in gynecologic oncology department, where there is another ongoing trial that overlaps with the objectives of this one, were asked to volunteer for the study. Among the clinician volunteers (including physicians, NPs and PAs), half were randomised into the intervention group, and the other half into a control group. Physicians, NPs and PAs in the intervention group were trained in using the SICG and received ongoing coaching from the study team. The control group provides usual care. In addition, because we hypothesise that volunteer clinicians would be different from non-volunteer clinicians and would therefore have patients with different outcomes than the patients of their volunteer-colleagues, we are enrolling the patients of a third group of clinicians who did not volunteer for the study and who also provide usual care (see figure 2 for the study design).

\section{Participant recruitment}

\section{Clinicians}

All medical oncologists who see patients at least one half day a week are eligible to participate. Study staff recruited and enrolled clinicians at disease centre meetings, by email and in person. Oncologists who declined participation received an email notifying them that their patients will be invited to participate. All volunteers received a $\$ 150$ gift card.

NPs and PAs were also enrolled in the study. They were recruited and consented in the same fashion as the physicians, and also received a $\$ 150$ gift card for enrolment. In order to minimise the possibility of contamination if a patient sees clinicians from both arms of the study, enrolled NPs and PAs were enrolled in the study arm of their collaborating physicians. If an NP or PA works with both intervention arm physicians and either control arm or non-enrolled physicians, they were excluded from the study.

\section{Patients}

Only patients receiving their ongoing primary oncology care at DFCI are eligible for participation in the study. Enrolment criteria include: age >18 years, English-speaking, able to consent and complete periodic surveys, and also to identify a friend or family member willing to answer surveys. Since the trial relies intensively on the clinician's communication skills about a culturally complex topic, we feel that relying on interpreters, who do not have experience with these conversations, and who have been shown to be unreliable translators of emotionally difficult material, ${ }^{23}{ }^{24}$ might expose patients and their families to greater distress without separate testing to determine suitability. Therefore, all non-English-speaking patients were excluded from the study (see figure 3 for a visual representation of recruitment).

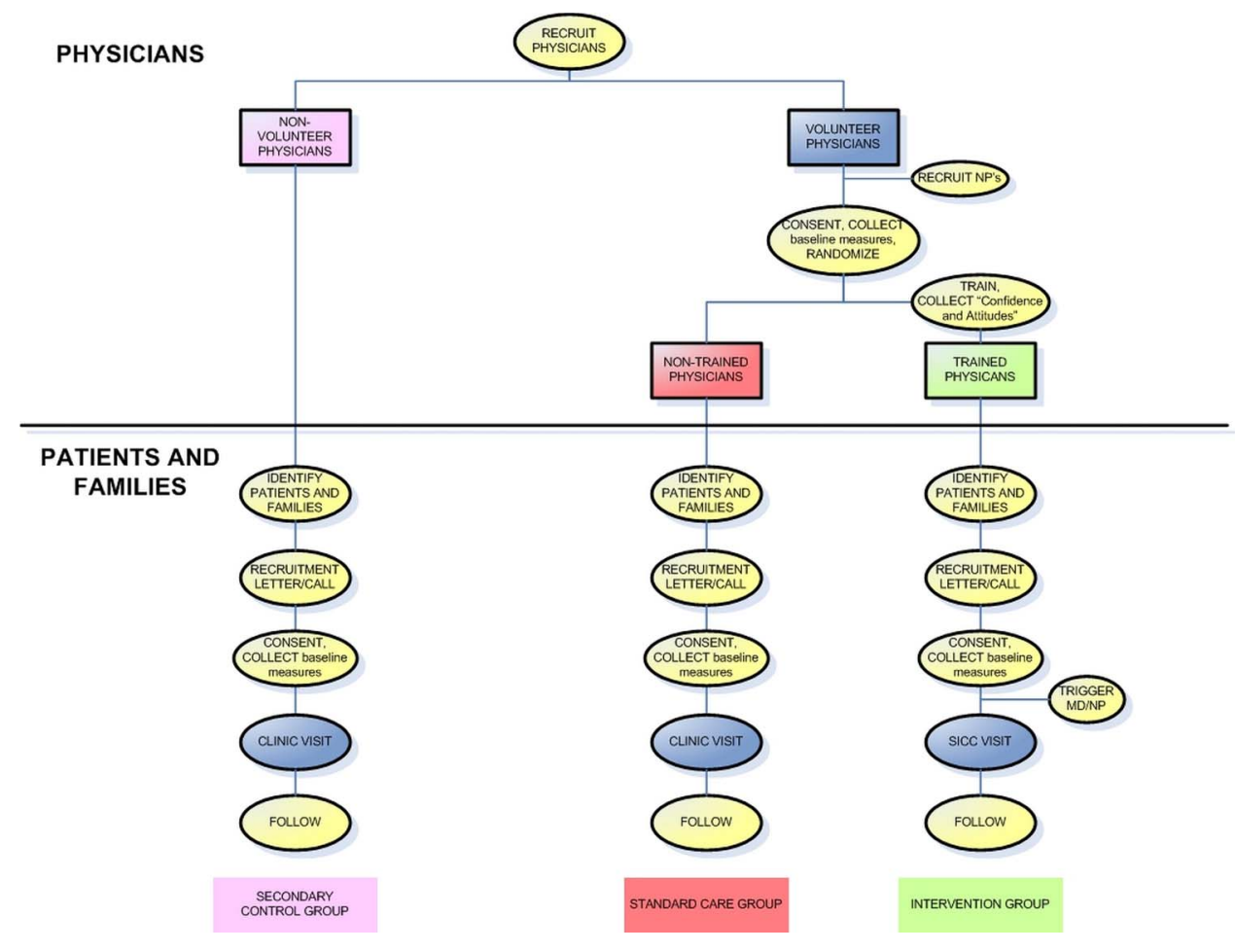

Figure 2 Research design. 
Figure 3 Patient/family recruitment and assessment.
PATIENTS

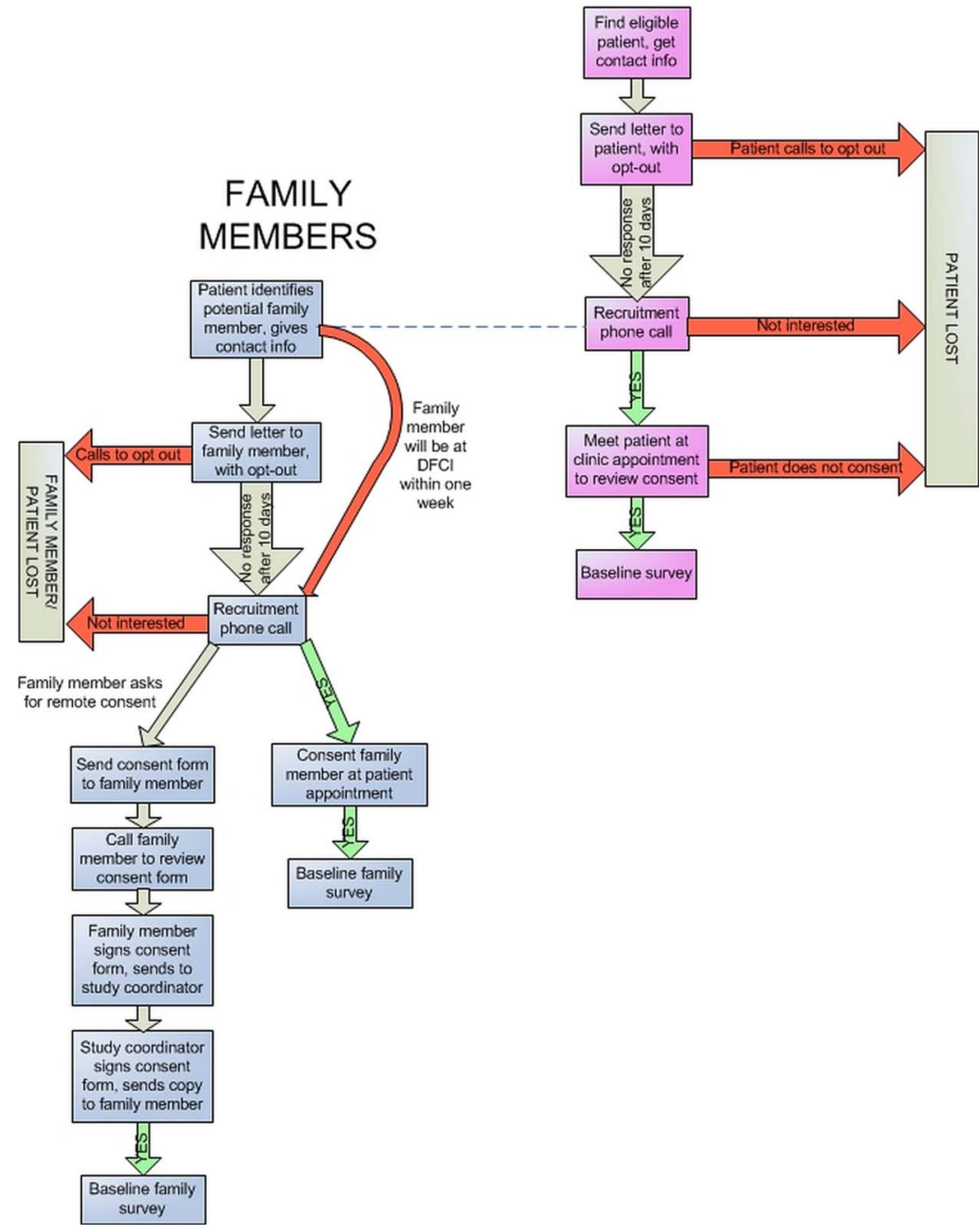

\section{Surrogates}

Patients identify a surrogate-a friend or family member - who is able to participate in the study. The friend or family member can consent remotely and is not required to attend clinic appointments with the patient but is asked to answer surveys. The friend or family member is required to be over 18, speak English, and have the ability to provide consent. If the identified surrogate does not wish to participate, then the patient is asked to suggest another individual. If no surrogate is identified, then the patient is ineligible for the study (see figure 3 for a visual representation of recruitment).

\section{Intervention}

The intervention is a multicomponent, structured communication intervention designed to (1) identify patients at high risk of death in the next year; (2) train clinicians to use the SICG to structure advanced care planning discussion with patients; (3) 'trigger' the oncology clinicians to have conversations using the guide with enrolled patients; (4) prepare patients for the conversation by providing them with a letter encouraging them to think about some of the topics raised in the Guide; (5) guide clinicians in conducting values and goals conversations; (6) document outcomes of the discussion in a structured format in the EMR and (7) provide patients with a Family Communication Guide to help them continue the discussion at home with their loved ones.

\section{Population identification}

To identify eligible patients, we use a 'No' answer to the question, 'Would you be surprised if this patient died in the next year?', if the patient meets other study requirements. The 'surprise question' is a validated single-item method for identifying patients at high risk of dying within a year among advanced cancer and dialysis patients. ${ }^{25}{ }^{26}$ A physician answering 'No' to the question 'Would I be surprised if this patient died in the next year?' confers an HR of death of 7.9 for patients with cancer. ${ }^{26}$ Every patient identified by a clinician with a 'No' response on the surprise question is invited to participate in the study by a letter with an opt-out card. If the opt-out card is not returned, a follow-up phone call is conducted to invite participation. 
Recruitment in the neuro-oncology clinic is conducted using the surprise question and via review of ICD-9 codes to identify any patients with a diagnosis of a cancer that has a high mortality risk (eg, glioblastoma multiforme); those patients are deemed eligible to be approached for consent after their fourth clinic visit.

\section{Training and coaching programme}

In order to minimise time commitment for clinicians, we designed a $2.5 \mathrm{~h}$ training programme for intervention clinicians to develop their competencies in using the $\operatorname{SICG}^{21}$ (table 1). The training begins with a brief didactic session $(<30 \mathrm{~min})$ on the evidence base for ACP discussions. A demonstration and discussion of the use of the SICG (approximately $30 \mathrm{~min}$ ) are followed by individualised practice by all participants, using roleplays with personalised feedback from attending palliative care physicians (more than $60 \mathrm{~min}$ ). Training sessions include 6-10 participants per session. Trained actors serve as standardised patients for both the demonstration and role-play. Clinicians learn how to introduce the conversation, including: (1) orienting patients to the conversation, (2) asking for permission to discuss desires for future care, (3) reassuring patients about continued treatment, (4) stating support for the patient and family and (5) emphasising that no decisions have to be made during the initial discussion. At the end of their patient conversations, clinicians are instructed to summarise and confirm the content of the conversation, and provide patients with a Family Communication Guide (figure 4) that can encourage further discussion of issues with family members. All intervention clinicians receive the Clinician Reference Guide, which summarises information provided in the training and offers guidance on challenging scenarios. The training concludes with a tutorial for clinicians on how to access and complete the EMR documentation module. Finding the time to conduct a conversation was a significant issue discussed in the training session. Clinicians were given leeway as to how to fit the conversation into their workday. We allowed clinicians to split the conversation between several visits, that is, that one or two questions would be addressed at each visit. We advised clinicians that we would continue to remind them to conduct the conversation until all questions were complete in the EMR documentation module.

After each intervention clinician's first SICG conversation with a patient, a physician member of the SICP team reviews the conversation, elicits feedback from the clinician, and offers suggestions. Intervention clinicians can also contact the SICP team in person, by paging and telephone or by email to discuss their conversations. Group or individual coaching sessions on difficulties with these conversations are similarly available from the SICP physicians, both for routine and challenging cases. In these sessions, the coach asks clinicians to identify

Table 1 Goals of the Serious IIIness Care Training Program

\begin{tabular}{|c|c|}
\hline \multicolumn{2}{|c|}{ By the end of the programme, learners will be able to demonstrate } \\
\hline Goal & Outcome \\
\hline \multicolumn{2}{|l|}{ 1. Communication skills } \\
\hline Use of silence, facilitating patient talk & $\begin{array}{l}\text { Allows silence before responding when patient is taking in } \\
\text { information or expressing emotion } \\
\text { Patient speaks }>50 \% \text { of time }\end{array}$ \\
\hline $\begin{array}{l}\text { Acknowledging and responding to patient/family } \\
\text { emotion }\end{array}$ & $\begin{array}{l}\text { Acknowledges difficult emotions during discussion } \\
\text { Responds to emotion with empathic comment or further } \\
\text { exploration } \\
\text { Avoids using information or premature reassurance to respond to } \\
\text { emotion }\end{array}$ \\
\hline Eliciting patient concerns & Encourages patient to express fears, worries, other concerns \\
\hline Assessing patient receptivity & $\begin{array}{l}\text { Accurately assesses patient's receptivity to receiving new } \\
\text { information and considering other options for treatment }\end{array}$ \\
\hline $\begin{array}{l}\text { Recognising appropriate time for exploration and for } \\
\text { making a recommendation }\end{array}$ & $\begin{array}{l}\text { Initiates conversation early in the course of illness, and when } \\
\text { prompted by disease progression or other clinical changes } \\
\text { Makes a recommendation based on an accurate assessment of } \\
\text { patient receptivity }\end{array}$ \\
\hline $\begin{array}{l}\text { Identifying key challenging scenarios in using the } \\
\text { SICG, and strategies for addressing them }\end{array}$ & $\begin{array}{l}\text { Describes concrete strategies for dealing with crying, anger, } \\
\text { denial, and avoidance }\end{array}$ \\
\hline $\begin{array}{l}\text { Use of follow-up questions to further explore } \\
\text { unclear or limited patient responses }\end{array}$ & $\begin{array}{l}\text { Asks follow-up questions when patient does not provide a full or } \\
\text { complete answer to SICG question }\end{array}$ \\
\hline \multicolumn{2}{|l|}{ 2. Mastery of SICG } \\
\hline Effective use of SICG & Clinician completes all elements of SICG \\
\hline Shares prognosis (as desired by patient) & $\begin{array}{l}\text { Gives prognosis in a range (days to weeks, weeks to months, etc), } \\
\text { with acknowledgement of uncertainty }\end{array}$ \\
\hline Documentation in ACP module & Documents critical information for colleagues in ACP module \\
\hline
\end{tabular}


Figure 4 Family communication guide (front page only).

\section{Talking about your illness with loved ones and caregivers}

This booklet can help you talk with your loved ones about your illness and the future. It is based on what you have already talked about with your doctor.

Talking about your illness with friends and family may not be easy, but it will help them understand what is important to you. It will also help them support you and your decisions.

Before you talk to your loved ones, think about when and where you want to talk. Choose a time and place when you feel relaxed. Be sure you have time to talk for a while. You can use the words

in this guide, or use your own words - whatever is easier for you.

problem areas and then facilitates discussion of approaches to the challenges presented, including use of role-play.

\section{Triggering}

Trained clinicians are triggered by the research staff to have the SICG discussion with enrolled patients in an upcoming visit in two ways: (1) an email is sent the day before the visit notifying the clinician that the study patient is due for an SICG conversation and (2) on the day of the visit, a packet with the study materials (SICG, Family Guide, and a Post-Conversation form) is placed with the face sheet that is given to clinicians before each patient visit. We collect data on the number of triggers it takes to complete the intended SICG conversations.

\section{Pre-visit letter}

In order to activate patients and ensure they are prepared for the conversation, intervention patients receive a pre-visit letter (figure 5), which introduces the SICG topics to consider prior to their conversation with their clinician.

\section{Serious IIIness Conversation Guide}

The seven key elements that should be addressed with patients are shown in figure 1. These include: illness understanding, decision-making and information preferences, prognostic disclosure, patient goals and fears, views on acceptable function and trade-offs, and desires for family involvement.

\section{Electronic medical record module documentation}

We developed a structured format for documentation that aligns with the seven items of the SICG in the ACP module of our EMR (figure 6). The module is designed to remind clinicians of the key elements of the discussion, to ease the burden of documentation, and to allow other clinicians easy access to the information elicited from the conversation. Each SICG question is mirrored in the module with a free text box and checkboxes in which to record responses. This module offers a single location for all information related to patient values and preferences, and allows interdisciplinary team members to review, enter and track preferences over time. All entries automatically include identification of the date, time and author of the documentation.

\section{Family guide}

After the conversation, the clinician gives patients a Family Guide that suggests an approach for discussing their illness and care preferences with their family.

\section{Outcome data collection}

The primary outcomes of the trial are patient receipt of goal-concordant care, and peacefulness at the end of life. Key process measures are acceptability of the SICG conversation to patients, acceptability of training to clinicians, number of triggers required to complete SICG, and frequency, timing, and quality of documentation of goals of care discussion. Secondary outcomes include: anxiety, depression, quality of life, therapeutic alliance, quality of communication, and quality of dying and death. We describe below how these outcomes will be 


\section{Talking with your doctor about the future}

At your next appointment, your doctor would like to talk with you about your illness, your goals and wishes, and planning for the future. This is an important part of the care we provide for all of our patients.

Our team likes to start talking about this when patients are doing okay. Your illness is serious but stable, so now is a good time to talk about what is ahead, and to do some planning for the future. Patients who think through what is important to them and what their wishes are often feel less anxious, more at peace, and more in control of their situation.

Before your next appointment

Please prepare for your visit by thinking about these things:

-What would you like to know about your illness and what is likely to be ahead?

- What kind of information would help you make decisions about your future?

- What is most important for you to have a good quality of life?

- What are you afraid of about your illness?

-What kinds of medical care do you not want?

- What do you think it would be like to share these thoughts with your family?

- If you haven't already identified a health care proxy, who would be able to fill that role?

Please bring to your visit:

- If you have a health care proxy form that is not on file at $\mathrm{DFCl}$, please bring a copy.

- If you have a living will or advance directive, please bring a copy.

If you don't have these documents or have questions about them, talk to your doctor.
Why is this important?

Thinking about and sharing your wishes will give you more control over the care you get. It will also help prepare your loved ones to make decisions for you if you can't make them at some point in the future.

Talking about the future won't change your ongoing care

Talking about the future won't change the plans we have made so far about your treatment, unless, of course, you want to. We will keep providing the best possible care to control your disease.

You may find it helpful to bring other people to your next appointment You can choose to bring the person who is your health care proxy or other family members to your next visit so they can be a part of the conversation. You can also bring your nurse practitioner, social worker, or chaplain if you like. Please let your doctor's office know if you would like to bring others to the appointment.

We understand that your wishes may change over time

This is the beginning of an ongoing conversation. We know that you may have other questions or concerns in the future. We will keep being here to support you and answer your questions so that you can make informed decisions.

If you have questions before your visit, please contact the study staff at 617-632-6055.

Figure 5 Pre-visit letter for patients.

measured. We plan to report outcomes in two phases: phase I will include key process measures and secondary outcomes and phase II will include the primary outcomes plus late secondary outcomes.

\section{Timing of assessments}

All study patients receive a baseline survey and then are surveyed every 2 months. For patients in the intervention arm, 1 week after the SICG conversation is complete, the patient is surveyed to assess their perception of the conversation, including its acceptability. Control patients are administered a parallel survey 2 months after enrolment, around the same time when patients in the intervention group would be likely to have an SICG conversation, based on pilot data prior to the randomised study. The control patients are asked about the number and content of their ACP or end-of-life discussions with their clinicians and families.

\section{Patient measures}

Table 2 lists the questionnaires and timing of administration for patients. Patients complete questionnaires by email, postal mail or phone. Demographic information includes age, gender, education, oncological disease, ethnicity and religion.

\section{Surrogate measures}

Table 3 lists the questionnaires and timing of administration for surrogates. We collect surrogate demographics including age, gender, education, ethnicity and religion. 


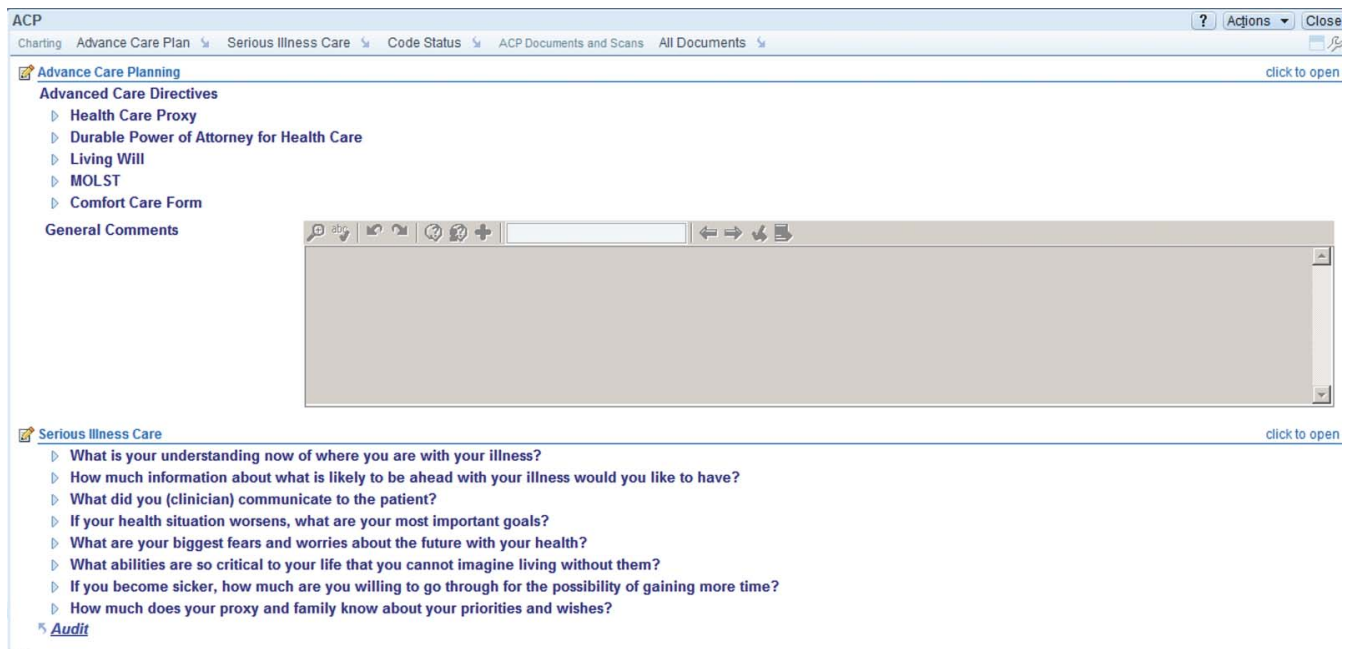

Figure 6 Electronic module of the Serious Illness Conversation Guide. (c) 2015 Epic Systems Corporation. Used with permission.

Friends/family members are surveyed every 2 months about the frequency and quality of communication with the patient about ACP and about their perception of the patient's life priorities using the Family Perception Scale. Six to 12 weeks after the patient's death, a social worker interviews the family member/friend on the phone using a modified form of the Quality of Dying and Death instrument (QODD). ${ }^{27-29}$ Additional questions from FAMCARE that address issues not covered in the QODD are included. The FAMCARE scale is a validated instrument to measure family satisfaction with advanced cancer care and can be administered to family members after the patient's death. ${ }^{30}$ We also collect the friend/family member's perceptions of the patient's achievement of self-identified goals using an adaptation of the Life Priorities measure. Prior to conducting the interview, which is audio-recorded, the social workers received a $1 \mathrm{~h}$ training from a bereavement specialist.

\section{Clinician measures}

Table 4 lists the questionnaires and timing of their administration for clinicians. At baseline, we record gender, profession (MD, NP or PA), disease centre, percentage clinical time, and years in practice, and administer questionnaires on clinician confidence in and attitudes about end-of-life conversations. The same questionnaire is administered following SICG training and will be administered at the conclusion of the study. Each time after a clinician is triggered to have a conversation, we ask them to complete a Post-Conversation Questionnaire. Clinicians provide information about prognostic communication, the length of the discussion, and whether they deviated from the SICG for any reason. For clinicians and patients who agree (a separate item on the consent form), a subset of conversations are audio-recorded. If the conversation did not take place, the same questionnaire asks clinicians to report their

Table 2 Patient measures and timing of administration

\begin{tabular}{|c|c|c|c|c|c|}
\hline \multirow[b]{2}{*}{ Domain } & \multirow[b]{2}{*}{ Measurement tool } & \multirow[b]{2}{*}{ Validated } & \multicolumn{3}{|c|}{ Timing of administration } \\
\hline & & & $\begin{array}{l}\text { Base } \\
\text { line }\end{array}$ & $\begin{array}{l}\text { After first } \\
\text { conversation or } \\
2 \text { months after } \\
\text { enrolment }\end{array}$ & $\begin{array}{l}\text { Every } \\
2 \text { months }\end{array}$ \\
\hline Demographics & & $\checkmark$ & $\checkmark$ & & \\
\hline Patient acceptability & 'Patient Acceptability' & & & $\checkmark$ & \\
\hline Quality of life & SF-12 & $\checkmark$ & $\checkmark$ & $\checkmark$ & $\checkmark$ \\
\hline Peacefulness & PEACE Questionnaire & $\checkmark$ & $\checkmark$ & $\checkmark$ & $\checkmark$ \\
\hline Life priorities & Purpose designed & & & $\checkmark$ & $\checkmark$ \\
\hline Goals of treatment & $\begin{array}{l}\text { Dichotomized choice question from } \\
\text { SUPPORT study }\end{array}$ & $\checkmark$ & $\checkmark$ & $\checkmark$ & $\checkmark$ \\
\hline Therapeutic alliance & Modified Human Connection Scale & $\checkmark$ & $\checkmark$ & $\checkmark$ & $\checkmark$ \\
\hline Anxiety & Generalized Anxiety Disorder (GAD 7) & $\checkmark$ & $\checkmark$ & $\checkmark$ & $\checkmark$ \\
\hline Depression & Patient Health Questionnaire (PHQ-9) & $\checkmark$ & $\checkmark$ & $\checkmark$ & $\checkmark$ \\
\hline \multirow[t]{2}{*}{$\begin{array}{l}\text { Quality of } \\
\text { communication }\end{array}$} & $\begin{array}{l}\text { Quality of Communication with } \\
\text { Clinician-Engelberg }\end{array}$ & $\checkmark$ & $\checkmark$ & $\checkmark$ & $\checkmark$ \\
\hline & 'Quality of Communication with Family' & & $\checkmark$ & $\checkmark$ & $\checkmark$ \\
\hline
\end{tabular}


Table 3 Surrogate measures and timing of administration

\begin{tabular}{|c|c|c|c|c|c|}
\hline \multirow[b]{2}{*}{ Domain } & \multirow[b]{2}{*}{ Measurement Tool } & \multicolumn{4}{|c|}{ Timing of administration } \\
\hline & & $\begin{array}{l}\text { Base } \\
\text { line }\end{array}$ & $\begin{array}{l}\text { After first } \\
\text { conversation or } \\
2 \text { months after } \\
\text { enrolment }\end{array}$ & $\begin{array}{l}\text { Every } \\
2 \text { months }\end{array}$ & $\begin{array}{l}\text { Eight weeks } \\
\text { after patient } \\
\text { death }\end{array}$ \\
\hline Demographics & & $\checkmark$ & & & \\
\hline $\begin{array}{l}\text { Quality of } \\
\text { communication }\end{array}$ & $\begin{array}{l}\text { 'Quality of Communication with } \\
\text { Family' }\end{array}$ & $\checkmark$ & $\checkmark$ & $\checkmark$ & \\
\hline Quality of death & Quality of Dying and Death (Curtis) & & & & $\checkmark$ \\
\hline
\end{tabular}

reasons for deferring the discussion. Clinician data also include how often we are emailed and paged for coaching by clinicians, as well as the number of one-on-one or group coaching sessions attended.

\section{Process measures}

Table 5 describes the process measures tracked in the study. We conduct a chart review to assess frequency, timing, and quality of conversations on patients who die. We will count the number of intervention and control patients with goals-of-care conversations documented in the EMR, the extent and retrievability of documentation in the EMR (eg, is the information in the Advance Care Planning Section of the EMR, or included in a progress or other note). In addition, the timing of conversations for intervention and control patients will be reported relative to time of death. Finally, we will count the number of key elements of goals-of-care discussion (based on best practices) documented in the EMR.

\section{Evaluation of goal concordance}

We could not identify a 'gold standard' for measurement of concordance between the patient's wishes and the care provided at the end of life; validated measures of this critical construct do not exist. ${ }^{31-36}$ Thus, we extensively reviewed existing literature on the topic, interviewed patients, and used this information to design a 'Life Priorities' survey for patients and a 'Family Perceptions' survey for identified surrogates. The Life Priorities survey evaluates patient priorities before death and the extent to which those priorities are subsequently achieved. The Family Perception survey assesses the extent to which the identified surrogate understands the patient's priorities while the patient is alive, and the extent to which those priorities were achieved in the last week and last 3 months of the patient's life, and also asks specific questions about the care received in the months, weeks and days before death. The last patientreported Life Priorities survey before death will be used as the primary outcome. We have included two additional validated questions in the patient surveys, and we will compare these responses to those from our Life Priorities Scale to evaluate the validity of our measure. ${ }^{37}$

To determine the extent to which patients receive care that is consistent with their top life priorities in the last 3 months and last week of life, we will compare the patient's top three ranked life priorities to the patient's care experience at the end of life using family perception of patient's achievement of goals and medical record review. Using that information and the scoring system of $0,1,2$ or 3 corresponding to the number of these top three goals that are met at the end of life, a team of independent, blinded physicians will determine the extent of goal concordance.

\section{Peacefulness}

The validated PEACE scale, or the 'Peace, equanimity and acceptance in the cancer experience' scale, will be used to measure patient peacefulness at the end of life. The PEACE instrument contains two subscales, 'Peaceful acceptance of Illness' and 'Struggle with Illness'. The 'Peaceful acceptance of Illness' subscale will be used as the main outcome. ${ }^{38}$

\section{Secondary outcomes}

We examine patient anxiety using the validated Generalized Anxiety Disorder (GAD-7) ${ }^{39} 40$ and evaluate depression using the validated Patient Health

Table 4 Clinician measures and timing of administration

\begin{tabular}{|c|c|c|c|c|c|}
\hline \multirow[b]{2}{*}{ Domain } & \multirow[b]{2}{*}{ Measurement tool } & \multicolumn{4}{|c|}{ Timing of administration } \\
\hline & & Base line & After training & After first conversation & End of study \\
\hline Attitudes & Clinician Attitudes Survey & $\checkmark$ & $\checkmark$ & & $\checkmark$ \\
\hline Confidence & Clinician Confidence Survey & $\checkmark$ & $\checkmark$ & & $\checkmark$ \\
\hline Acceptability & Clinician Acceptability Survey & & & $\checkmark$ & $\checkmark$ \\
\hline Prognostic evaluation & Post-conversation Form & \multicolumn{4}{|c|}{ After every conversation } \\
\hline
\end{tabular}


Table 5 Process measures: frequency, timing and quality of conversations

\begin{tabular}{ll}
\hline Outcome & Measure \\
\hline Frequency & $\begin{array}{l}\text { Chart review of outpatient oncology progress } \\
\text { notes to determine the percentage of } \\
\text { intervention and control patients who had } \\
\text { documentation of goals of care before death }\end{array}$ \\
Timing & $\begin{array}{l}\text { Chart review of outpatient oncology progress } \\
\text { notes to determine the timing of the first } \\
\text { documented goals-of-care conversation } \\
\text { before death in intervention and control } \\
\text { patients } \\
\text { Chart review of outpatient oncology } \\
\text { documentation to assess retrievability } \\
\text { (Advance Care Planning Section of EMR vs } \\
\text { progress notes vs other locations) and to } \\
\text { compare the number of key elements } \\
\text { addressed in documented goals of care } \\
\text { conversations in intervention and control } \\
\text { patients }\end{array}$ \\
\hline EMR, electronic medical record.
\end{tabular}

Questionnaire (PHQ-9). ${ }^{41}$ We measure therapeutic alliance using the Human Connection Scale. ${ }^{42}$ The Human Connection Scale is a valid and reliable measure of therapeutic alliance between patients with advanced cancer and their physicians. ${ }^{42}$ We assess patient perception of the quality of communication with their clinician $^{32}{ }^{43}$ using the 'Quality of Communication' (QOC) scale, a 13-item validated questionnaire that measures the quality of end-of-life communication in two scales: a six-item 'general communication skills' scale and a seven-item 'communication about end-of-life care' scale. ${ }^{44}$ We measure quality of life and general physical health function by the SF-12 V2 health survey. ${ }^{45}$ It consists of 12 items and provides scores for both overall physical and mental health. We use the Brief R-COPE, a 6-item measure that assesses positive religious (PRC)/ spiritual methods of coping and dealing with life stressors, and negative religious (NRC)/spiritual methods of coping that are representative of the religious struggle when dealing with life stressors. ${ }^{46}$

\section{Sample size}

Power calculations were performed for the two primary aims of the study: comparison of enhanced goal-consistent care and comparison of the PEACE measure across the randomised arms. In order to ensure an overall $5 \%$ type I error rate for the two primary aims, we will use a $2.5 \%$ type I error rate for each of the two primary hypotheses. Both aims are based on patient death; we expect to follow each patient for at least 1 year from randomisation, or until death if $<1$ year from randomisation. The power calculations below are based on having 200 evaluable patients per arm. We allow for $6 \%$ unevaluability due to patient dropout, meaning a total of 426 patients (213 per group) will be accrued at an estimated accrual rate of 200 patients per year. Given that each patient must be followed for at least 1 year from randomisation, we expect the study to be open for at least 3 years.

\section{Primary aim 1}

Enhanced goal-consistent care. The outcome for a patient will be a score of $0,1,2$ or 3 corresponding to the number of the top three high priority goals that are met in the last week and 3 months of life. Based on previous data, the SD of 'number of high priority goals met' is conservatively estimated to be 1.35 , and we expect the average 'number of high priority goals met' to be at least 0.6 point higher with the SICG (a clinically important increase based on prior studies). The two arms will be compared using a robust generalised estimating equations (GEE) Wilcoxon rank-sum-type score test ${ }^{47}$ for ordinal categorical data; this approach does not assume normality of the outcome, and accounts for a possible cluster effect of patients within clinician. Using the GEE Wilcoxon rank-sum score test with a two-sided type I error rate of $2.5 \%$ and 200 evaluable patients per arm, we have over $80 \%$ power to detect an average 0.6 point higher average score in the SICG arm. In this power calculation, an intracluster (clinician) correlation coefficient (ICC) of approximately 0.1 has been assumed, as is (conservatively) commonly used in this type of cluster randomisation study. ${ }^{48}$

\section{Primary aim 2}

PEACE scale. From previous data, ${ }^{38}$ the $\mathrm{SD}$ of the 'Peaceful acceptance subscale' is conservatively estimated to be 3.3 ('conservatively', since this is the largest SD found in Mack $e t a l^{88}$ ), and we expect the average score to be at least 1.3 points higher with the intervention (again, a clinically important increase based on prior studies). The two arms will be compared using the GEE Wilcoxon rank-sum score test accounting for clustering of patients within clinician. Using a GEE Wilcoxon rank-sum score test with a two-sided type I error rate of $2.5 \%$ and 200 evaluable patients per arm, we have over $80 \%$ power to detect a 1.3-point higher average 'Peaceful acceptance subscale' in the intervention arm, assuming the $\mathrm{ICC}=0.1$.

\section{Interim analysis/early stopping rules}

For primary aims 1 and 2, the trial will be monitored for possible early stopping due to a large intervention effect using a Haybittle-Peto approach. ${ }^{49}$ Specifically, one interim GEE Wilcoxon rank-sum score test will be performed after approximately $50 \%$ of the total patients are enrolled, which should occur approximately 6 months after baseline and with 213 patients. In order to stop the study at this point in favour of the SICG arm, the $\mathrm{p}$ value for GEE Wilcoxon rank-sum score test must be $<0.001$. The power calculations above take into account the possibility of one interim analysis. The stopping rule for this study will be used as a guideline rather than as a hard-and-fast rule. Any final decision will also consider 
additional end points such as differences in complications and process measures. If the findings of the interim analysis are in favour of stopping the trial due to beneficial effect of the SICG, the research study will be stopped. We note here that we used a simpler conservative interim analysis approach ${ }^{49}$ because the less conservative approaches ${ }^{50}$ require independent increments of data; however, the data will not likely come to us in independent increments because of the cluster randomised design. Further, as we have chosen a conservative interim analysis approach, the one interim analysis can be performed at any time during the study, and the stopping rule of $p<0.001$ still applies.

\section{Randomisation}

The oncologists are stratified by disease centre or satellite facility. The strata correspond to the disease centres (breast, gastrointestinal, genitourinary, leukaemia, lymphoma, thoracic, sarcoma, head \& neck, neuro-oncology) and two satellite facilities. The smallest disease centre, melanoma, was not randomised because cross contamination would be likely due to its small size; it served as a pilot site. Clusters are organisational units of clinicians within a disease centre; a typical cluster would have one NP and 2-3 physicians, although the numbers of particular clinicians vary across disease centres; randomisation was carried out by cluster.

Within strata, one-half of the clusters were randomised to the intervention and one-half of the clusters were randomised to standard care. The cluster is randomly assigned to intervention or control based on a random number drawn in SAS. The GEE statistical methods described in the power calculations take into account the stratification (disease centre) and clustering (clinicians) variables in the analysis.

\section{Blinding}

For the telephone bereavement interviews, social workers who are not involved in the study conduct the interviews and are blinded to the study arm of the patient and surrogate (intervention vs control).

\section{Planned analytic approach}

The objective of this study is to evaluate whether, for patients with cancer, the SICP is better than usual care at helping patients achieve healthcare goals and quality of life in the setting of serious illnesses. The design is a cluster-randomised controlled trial in which patients are nested within clinician clusters. One-half of the oncology clinicians are randomised to the SICP and one-half of the oncology clinicians are randomised to standard care. Since this is a randomised trial with stratification by disease centre prior to randomisation, we expect the demographic characteristics and other possible confounders (age, disease severity, etc) to be similar in the two groups. However, since randomisation is at the clusterlevel and there are 50 total clusters, there is a slightly greater chance that characteristics will not balance out than if randomisation was done at the patient level. Prior to evaluating the effect of the intervention, the demographic characteristics of test arms will be compared by the Rao-Scott $\chi^{2}$ (accounting for stratification by disease centre and clustering by oncology clinician) for discrete variables, and by the GEE Wilcoxon rank-sum score test for ordered categorical variables and continuous variables (accounting for stratification by disease centre and clustering by oncology clinician). ${ }^{47}$ If, as expected, there are no differences in demographic or other confounders, the primary outcomes across arms will be compared using the GEE Wilcoxon rank-sum score test for ordered categorical variables and continuous variables (accounting for stratification by disease centre and clustering by oncologist). ${ }^{47}$

If baseline differences are found between the two arms with respect to important confounders (such as age, race, comorbidities), the GEE Wilcoxon rank-sum test will incorporate propensity weights. In particular, to conduct the propensity score adjustment, we will use a logistic regression model to calculate the propensity (probability) of being in the intervention or control groups based on all possible confounders, and then weight each participant in the GEE Wilcoxon rank-sum score test based on the inverse propensity of being in one of the two treatment groups. ${ }^{51}$ This study evaluates the impact of the entire intervention, and will not address which components of this multicomponent programme are effective.

\section{ETHICS AND DISSEMINATION \\ Ethical review}

All participants provide informed consent and may withdraw at any time without impacting their treatment or relationship with their clinical team. An approved protocol to monitor for adverse events is followed and additional support for patients with symptoms of psychological distress is available through the Palliative Care and Psychosocial Oncology clinical programmes. Additionally, we monitor patient responses to the PHQ-9 measure of depression whenever these responses are collected. If the patient answers 'More than half the days' or 'Nearly every day' to the question, 'Thoughts that you would be better off dead, or of hurting yourself', or if the patient responses indicate severe depression (PHQ-9 score of 20 or greater), we immediately notify the patient's NP or physician by pager or phone. We also monitor the GAD-7 measure of anxiety and the pain score to screen for the following indicators of patient distress: severe anxiety (GAD-7 score of 15 or greater), and severe pain (level of 8 or greater). When any of these conditions are met, we notify the patient's NP or physician by email within $24 \mathrm{~h}$.

\section{Relevance and dissemination}

The design of the SICP addresses critical deficiencies in the conduct of end-of-life conversations. The SICP, if successful, provides a simple, scalable structure to 
support oncology physicians, NPs and PAs in conducting state-of-the-art serious illness conversations with a broad array of oncology outpatients at a high risk of dying within a year. We do not know the generalisability to other populations of seriously ill patients, such as those with congestive heart failure or chronic obstructive pulmonary disease. Likewise, we are limited to analysing the data of the patients who agreed to participate in the trial and cannot characterise patients who opted out, who might be less willing to discuss these issues.

The Conversation Guide, while not comprehensive, has been designed to balance brevity to enhance scalability, while at the same time addressing the issues that matter most to patients. The length of the clinician training $(2.5 \mathrm{~h})$ was chosen as an acceptable amount of time that would be feasible for many healthcare systems to adopt, if successful. We anticipate that clinician training will improve the quality of these conversations, and that enhancing clinician confidence, knowledge, and skill, the lack of which are known barriers to ACP, will increase the frequency of effective serious illness conversations. By triggering the conversations early in the course of the illness, our goal is for patients with serious illness and their families to be able to consider decisions about their lives and choices about medical care with adequate time for reflection and discussion. The design of the EMR module creates a repository of information about patient values and goals, reinforces use of the SICG, reduces time needed to record information, and communicates patient goals and values in a readily accessible place in the EMR that is easily retrievable by other clinicians, especially in an emergency.

Our ultimate aim is to determine if we can provide a proven means for transforming end-of-life care through an innovative model that enables patients with serious illness and non-palliative care clinicians to discuss preferences for end-of-life care more often, earlier, and more effectively. We believe that developing scalable models for improving serious illness conversations will contribute to better alignment of healthcare with the preferences of oncology patients, and eventual extension to other patient populations and care settings.

\section{Author affiliations \\ ${ }^{1}$ Harvard Medical School, Boston, Massachusetts, USA \\ 2Department of Psychosocial Oncology and Palliative Care, Dana-Farber Cancer Institute, Boston, Massachusetts, USA \\ ${ }^{3}$ Ariadne Labs at Brigham and Women's Hospital and the Harvard T. H. Chan School of Public Health, Boston, Massachusetts, USA \\ ${ }^{4}$ Harvard Medical School Center for Palliative Care, Boston, Massachusetts, USA \\ ${ }^{5}$ Johns Hopkins University School of Medicine, Baltimore, Maryland, USA \\ ${ }^{6}$ University of California San Francisco, San Francisco, California, USA}

Twitter Follow RACHELLE E. BERNACKI at @rbernack

Contributors SDB is the Director and Principal Investigator of the Serious Illness Care Program. RB is the Project Director and has been centrally involved in all aspects of the trial, including the development of the study protocol. MH is study coordinator and has the primary responsibility of coordinating development of the study materials, and contributed to the development of the protocol. JV, GS and JP contributed to the development of the materials as well as the protocol. SL is the primary biostatistician for the study. AAG and SDB obtained funds for this trial; AAG also serves as a senior advisor and co-investigator for the project. All authors reviewed and approved the manuscript. AAG receives royalties from multiple publishers and media outlets for essays, books, and films on improvement of healthcare, including those on end-of-life care.

Funding This work was supported by the Charina Endowment Fund, Partners Healthcare, and the Margaret T. Morris Foundation. RB is supported by Health Resources Services Administration grant K01HP2046.

Competing interests None declared.

Ethics approval The study was approved by the Dana-Farber Cancer Institute Institutional Review Board.

Provenance and peer review Not commissioned; externally peer reviewed.

Open Access This is an Open Access article distributed in accordance with the Creative Commons Attribution Non Commercial (CC BY-NC 4.0) license, which permits others to distribute, remix, adapt, build upon this work noncommercially, and license their derivative works on different terms, provided the original work is properly cited and the use is non-commercial. See: http:// creativecommons.org/licenses/by-nc/4.0/

\section{REFERENCES}

1. Institute of Medicine. Dying in America: improving quality and honoring individual preferences near end of life. Washington, DC, 2014

2. Wright AA, Mack JW, Kritek PA, et al. Influence of patients' preferences and treatment site on cancer patients' end-of-life care. Cancer 2010;116:4656-63.

3. Detering KM, Hancock AD, Reade MC, et al. The impact of advance care planning on end of life care in elderly patients: randomised controlled trial. BMJ 2010;340:c1345

4. Temel JS, Greer JA, Muzikansky A, et al. Early palliative care for patients with metastatic non-small-cell lung cancer. $N$ Engl $J$ Med 2010;363:733-42

5. Mack JW, Cronin A, Taback N, et al. End-of-life care discussions among patients with advanced cancer: a cohort study. Ann Intern Med 2012;156:204-10.

6. Curtis JR, Patrick DL, Caldwell ES, et al. Why don't patients and physicians talk about end-of-life care? Barriers to communication for patients with acquired immunodeficiency syndrome and their primary care clinicians. Arch Intern Med 2000;160:1690-6.

7. Anderson WG, Chase R, Pantilat SZ, et al. Code status discussions between attending hospitalist physicians and medical patients at hospital admission. J Gen Intern Med 2011;26:359-66.

8. Hagerty RG, Butow PN, Ellis PA, et al. Cancer patient preferences for communication of prognosis in the metastatic setting. J Clin Oncol 2004;22:1721-30.

9. Smith AK, McCarthy EP, Paulk E, et al. Racial and ethnic differences in advance care planning among patients with cancer: impact of terminal illness acknowledgment, religiousness, and treatment preferences. J Clin Oncol 2008;26:4131-7.

10. Buss MK, Lessen DS, Sullivan AM, et al. Hematology/oncology fellows' training in palliative care: results of a national survey. Cancer 2011;117:4304-11.

11. Block SD. Medical education in end-of-life care: the status of reform. $J$ Palliat Med 2002;5:243-8.

12. Fallowfield L, Jenkins V, Farewell V, et al. Efficacy of a Cancer Research UK communication skills training model for oncologists: a randomised controlled trial. Lancet 2002;359:650-6.

13. Back AL, Arnold RM, Baile WF, et al. Faculty development to change the paradigm of communication skills teaching in oncology. $J$ Clin Oncol 2009;27:1137-41.

14. Sullivan AM, Lakoma MD, Billings JA, et al. Teaching and learning end-of-life care: evaluation of a faculty development program in palliative care. Acad Med 2005;80:657-68.

15. Sullivan AM, Lakoma MD, Billings JA, et al. Creating enduring change: demonstrating the long-term impact of a faculty development program in palliative care. J Gen Intern Med 2006;21:907-14.

16. Rao JK, Anderson LA, Inui TS, et al. Communication interventions make a difference in conversations between physicians and patients: a systematic review of the evidence. Med Care 2007;45:340-9. 
17. Fellowes D, Wilkinson S, Moore P. Communication skills training for health care professionals working with cancer patients, their families and/or carers. Cochrane Database Syst Rev 2004;(2):CD003751.

18. Jacobsen J, Jackson V, Dahlin C, et al. Components of early outpatient palliative care consultation in patients with metastatic nonsmall cell lung cancer. J Palliat Med 2011;14:459-64.

19. Lupu D. Estimate of current hospice and palliative medicine physician workforce shortage. J Pain Symptom Manage 2010;40:899-911.

20. Block SD, Billings JA. A need for scalable outpatient palliative care interventions. Lancet 2014;383:1699-700.

21. Bernacki RE, Block SD, American College of Physicians High Value Care Task Force. Communication about serious illness care goals: a review and synthesis of best practices. JAMA Intern Med 2014;174:1994-2003.

22. Billings JA, Bernacki R. Strategic targeting of advance care planning interventions: the Goldilocks phenomenon. JAMA Intern Med 2014;174:620-4.

23. Brach C, Fraser I, Paez K. Crossing the language chasm. Health Aff (Millwood) 2005;24:424-34.

24. Thornton JD, Pham K, Engelberg RA, et al. Families with limited English proficiency receive less information and support in interpreted intensive care unit family conferences. Crit Care Med 2009;37:89-95.

25. Moss AH, Ganjoo J, Sharma S, et al. Utility of the "surprise" question to identify dialysis patients with high mortality. Clin J Am Soc Nephrol 2008;3:1379-84.

26. Moss AH, Lunney JR, Culp S, et al. Prognostic significance of the "surprise" question in cancer patients. J Palliat Med 2010;13:837-40.

27. Curtis JR, Patrick DL, Engelberg RA, et al. A measure of the quality of dying and death. Initial validation using after-death interviews with family members. J Pain Symptom Manage 2002;24:17-31.

28. Engelberg RA, Patrick DL, Curtis JR. Correspondence between patients' preferences and surrogates' understandings for dying and death. J Pain Symptom Manage 2005;30:498-509.

29. Patrick DL, Curtis JR, Engelberg RA, et al. Measuring and improving the quality of dying and death. Ann Intern Med 2003;139(5 Pt 2):410-15.

30. Kristjanson LJ. Validity and reliability testing of the FAMCARE Scale: measuring family satisfaction with advanced cancer care. Soc Sci Med 1993;36:693-701.

31. Steinhauser KE, Christakis NA, Clipp EC, et al. Factors considered important at the end of life by patients, family, physicians, and other care providers. JAMA 2000;284:2476-82.

32. Steinhauser KE, Clipp EC, McNeilly M, et al. In search of a good death: observations of patients, families, and providers. Ann Intern Med 2000;132:825-32.

33. Quill T, Norton S, Shah M, et al. What is most important for you to achieve? An analysis of patient responses when receiving palliative care consultation. J Palliat Med 2006;9:382-8.

34. Barnato AE, Llewellyn-Thomas HA, Peters EM, et al. Communication and decision making in cancer care: setting research priorities for decision support/patients' decision aids. Med Decis Making 2007;27:626-34.

35. Clayton J, Butow $\mathrm{P}$, Tattersall $\mathrm{M}$, et al. Asking questions can help: development and preliminary evaluation of a question prompt list for palliative care patients. Br J Cancer 2003;89:2069-77.

36. Mack JW, Weeks JC, Wright AA, et al. End-of-life discussions, goal attainment, and distress at the end of life: predictors and outcomes of receipt of care consistent with preferences. J Clin Oncol 2010;28:1203-8.

37. [No authors listed]. A controlled trial to improve care for seriously ill hospitalized patients. The study to understand prognoses and preferences for outcomes and risks of treatments (SUPPORT). The SUPPORT Principal Investigators. JAMA 1995;274:1591-8.

38. Mack JW, Nilsson M, Balboni T, et al. Peace, Equanimity, and Acceptance in the Cancer Experience (PEACE): validation of a scale to assess acceptance and struggle with terminal illness. Cancer 2008;112:2509-17.

39. Cameron IM, Crawford JR, Lawton K, et al. Psychometric comparison of PHQ-9 and HADS for measuring depression severity in primary care. Br J Gen Pract 2008;58:32-6.

40. Spitzer RL, Kroenke K, Williams JB, et al. A brief measure for assessing generalized anxiety disorder: the GAD-7. Arch Intern Med 2006;166:1092-7.

41. Martin A, Rief W, Klaiberg A, et al. Validity of the Brief Patient Health Questionnaire Mood Scale (PHQ-9) in the general population. Gen Hosp Psychiatry 2006;28:71-7.

42. Mack JW, Block SD, Nilsson M, et al. Measuring therapeutic alliance between oncologists and patients with advanced cancer: the Human Connection Scale. Cancer 2009;115:3302-11.

43. Wenrich MD, Curtis JR, Shannon SE, et al. Communicating with dying patients within the spectrum of medical care from terminal diagnosis to death. Arch Intern Med 2001;161:868-74.

44. Engelberg R, Downey L, Curtis JR. Psychometric characteristics of a quality of communication questionnaire assessing communication about end-of-life care. J Palliat Med 2006;9:1086-98.

45. Ware J Jr., Kosinski M, Keller SD. A 12-Item Short-Form Health Survey: construction of scales and preliminary tests of reliability and validity. Med Care 1996;34:220-33.

46. Pargament K, Feuille M, Burdzy D. The brief RCOPE: current psychometric status of a short measure of religious coping. Religions 2011;2:51-76.

47. Natarajan S, Lipsitz SR, Fitzmaurice GM, et al. An extension of the Wilcoxon Rank-Sum test for complex sample survey data. J R Stat Soc Ser C Appl Stat 2012;61:653-64.

48. Donner A, Klar N. Pitfalls of and controversies in cluster randomization trials. Am J Public Health 2004;94:416-22.

49. Haybittle JL. Repeated assessment of results in clinical trials of cancer treatment. Br J Radiol 1971;44:793-7.

50. O'Brien PC, Fleming TR. A multiple testing procedure for clinical trials. Biometrics 1979;35:549-56.

51. Robins JM, Hernan MA, Brumback B. Marginal structural models and causal inference in epidemiology. Epidemiology 2000;11:550-60. 